\title{
SIMULTANEOUS SOLUTION FOR THE MASSES OF THE PRINCIPAL PLANETS FROM ANALYSIS OF OPTICAL, RADAR, AND RADIO TRACKING DATA*
}

\author{
J. H. LIESKE, W. G. MELBOURNE, D. A. O'HANDLEY, \\ D. B. HOLDRIDGE, D. E. JOHNSON, and W. S. SINCLAIR \\ Jet Propulsion Laboratory, Pasadena, Calif., U.S.A.
}

\author{
(Presented at IAU Colloquium No. 9, 'The IAU System of \\ Astronomical Constants', Heidelberg, Germany, August 12-14, 1970.)
}

\begin{abstract}
The Jet Propulsion Laboratory has developed a set of computer programs known as the Solar System Data Processing System (SSDPS) which is employed in improving the ephemerides of the major planets and for improving the values of several associated astronomical constants. A group of solutions for the masses of the major planets, together with the AU and radii of Mercury, Venus, and Mars, is presented. These solutions based upon optical, radar, and spacecraft radio tracking data are preliminary. The relative power of radar and radio tracking data vis-à-vis purely optical data in a solution is shown. The problems which could arise by adopting solutions based upon a single data type are demonstrated.
\end{abstract}

\section{Introduction}

The Jet Propulsion Laboratory (JPL) has developed a set of computer programs known as the Solar System Data Processing System (SSDPS) which is employed in improving the ephemerides of the major planets and for obtaining improved values for several associated astronomical constants.

In the initial phase of ephemeris improvement at JPL, the time interval under consideration was from 1967 back to 1950. In the current phase of this effort, the time interval covers the period 1969 back to approximately 1910 .

The observational data set contains over 37600 observations of the major planets. These observations are taken from transit-circle measurements of all the planets except Pluto for which the observations are photographic. Radar time-delay measurements for Mercury, Venus, and Mars taken over the interval 1964-69 are included. Spacecraft radio tracking data represents a significant new data type which is included in the analysis. The spacecraft data currently consist of range and Doppler measurements obtained during the encounter portion of the Mariner 5 Venus flyby in October 1967.

In this preliminary analysis, an attempt has been made to solve for a set of 75 parameters. These include the elements of the 9 major planets, the rectangular coordinates and velocity components of the Mariner 5 spacecraft, the right ascension and declination limb biases for Mercury and Venus, the radii of Mercury, Venus, and Mars, the astronomical unit (AU), and the masses of the major planets except for the

* This paper presents the results of one phase of research carried out at the Jet Propulsion Laboratory, California Institute of Technology, under Contract No. NAS 7-100, sponsored by the National Aeronautics and Space Administration. 
Earth-Moon barycenter and Mars. The latter two masses have been determined from Ranger and Mariner spacecraft radio tracking data which are not included in this preliminary analysis. In the future, all significant spacecraft data will be included in a gravitationally consistent solution. Admittedly the equator and equinox, the constant of precession, catalogue corrections, and other parameters can be differentially corrected. However, experience has indicated that although reasonable corrections have been determined, the application of these corrections is not clear because of the degree to which these physical constants are imbedded in the original reduction of the star catalogues and planetary observations.

The system of planetary masses presented here should be considered as preliminary. The important result of this analysis is that significant variations in the solutions for the masses and other constants can result from the addition of either radar or spacecraft data to the basic set of optical observations. Before a definitive set of constants can be obtained, it will be necessary to iterate the solutions currently available and, in addition, to introduce more spacecraft and radar data and to increase the optical data arc. At this time it would appear that definitive planetary masses can be obtained from spacecraft radio tracking data near encounter, but for the planet Mercury or the outer planets, for which spacecraft data are not available, one can merely bound the values of the masses until additional information is available.

\section{Analysis Program}

The Solar System Data Processing System (SSDPS) consists of a set of computer programs (containing over 300000 coded instructions) arranged in modular form, which is designed to process and analyze planetary and spacecraft observations for the purpose of improving planetary ephemerides and astronomical constants.

The system was originally developed by C. L. Lawson of JPL in 1966, and a flow diagram of its current configuration is depicted in Figure 1. A geocentric lunar ephemeris (available on magnetic tape), together with the initial positions, velocities and relevant astronomical constants, is input to the program PLINT which integrates numerically the differential equations (of order 54) describing the heliocentric motion of the 9 principal planets. The program employs a second-sum Gauss-Jackson method of integration retaining up to the twelfth backward differences in the accelerations. A half-day step size is used for all planets; predictor and corrector formulas are employed in obtaining the positions of Mercury and Venus while the predictor formulas alone are used for the other planets. The Earth and Moon are gravitationally treated as separate bodies, but, for the sake of convenience, the coordinates of the Earth-Moon barycenter are integrated rather than those of the Earth alone; the lunar effects are obtained from magnetic tape. The differential equations of motion include the effects of general relativity. A spherically symmetric isotropic line element which parameterizes the gravitational field of the Sun by the Eddington-Robertson formalism (Robertson, 1962; Eddington, 1960) is employed. Nominally, the parameters of this line element are set equal to unity, this having the effect of reducing the gravita- 


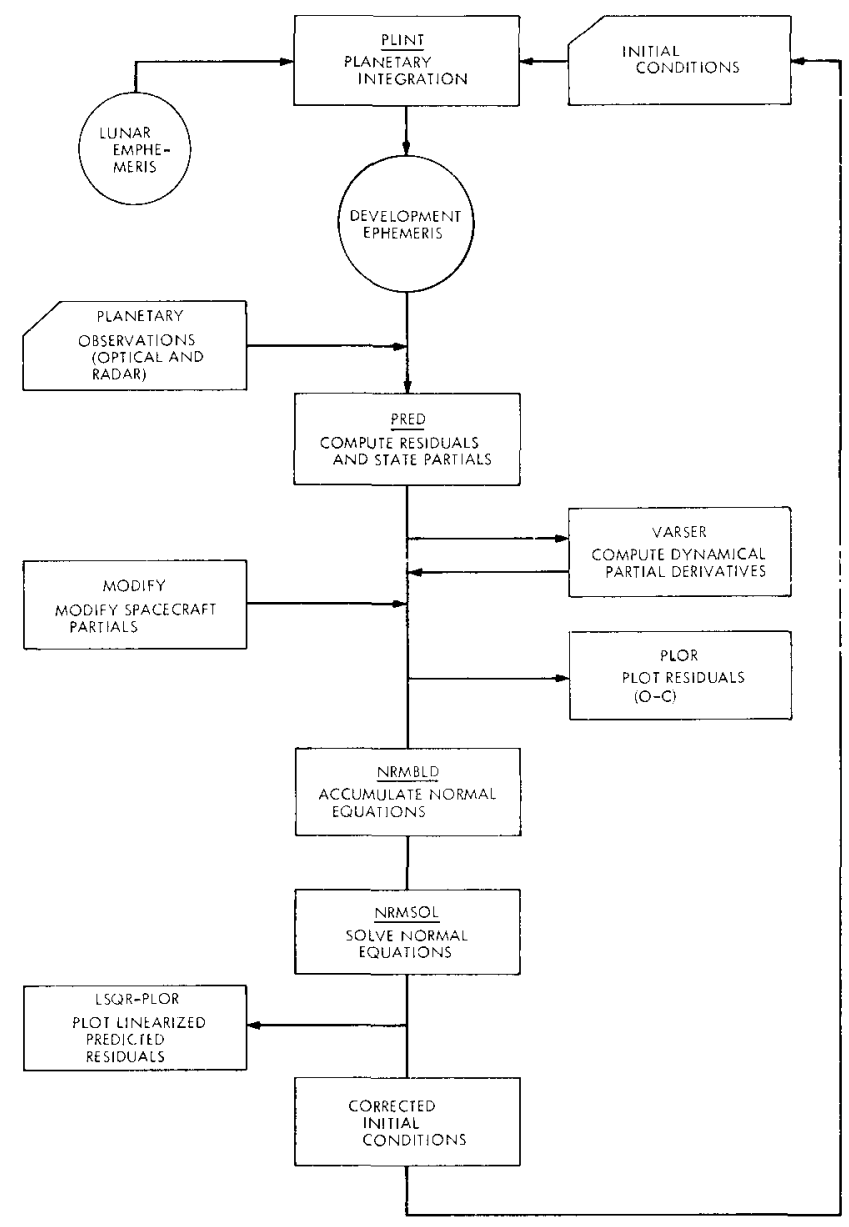

Fig. 1. Solar System Data Processing System (SSDPS) flow diagram.

tional field to the Schwarzschild solution of Einstein's equations in isotropic coordinates. The ephemerides resulting from the numerical integration are then output onto magnetic tape for use in further analysis or in other problems where precise ephemerides are required.

To analyze the available planetary observations, the optical and radar data are input to a program link called PRED. The PRED routines compute the observables from the previously available ephemeris tape and form an array containing the observed and computed quantities, together with the Eckert-Brouwer Set III (Eckert and Brouwer, 1937) partial derivatives describing the effects of small changes in the planetary positions. The results are put on magnetic tape for subsequent processing.

If partial derivatives describing the effects of changes in dynamical parameters (e.g. planetary masses, solar oblateness, relativistic parameters) upon the data are required, then a set of programs called VARSER is employed. In these routines the 
partial derivatives of the data with respect to the dynamical parameters are computed by means of evaluating Fourier series or Chebyshev polynomials. The Fourier/ Chebyshev series are obtained by generating first-order general theories (Broucke and Garthwaite, 1969; Broucke, 1969) which analytically describe the effects of the dynamical parameters. By a suitable choice of the reference orbit (i.e., an ellipse osculating at the adopted ephemeris epoch JD 2440800.5), the resultant series may be regarded as analytic expressions for the partial derivatives of the coordinates with respect to a dynamical parameter. The partial derivatives are then combined onto a new tape with the previously computed results from PRED and are available for further processing.

Spacecraft data when received from the tracking stations must be preprocessed through a series of programs in order to be compatible with this system of programs. Interface programs, called POSTRE and MODIFY, are used respectively to alter the format of the data and map the partial derivatives of the spacecraft data from the epoch near encounter to the adopted ephemeris epoch.

At this stage the planetary and/or spacecraft data and partial derivatives are accumulated by NRMBLD into normal equations for further analysis.

In the program NRMSOL, the normal equations are then solved in order to obtain corrections to the various quantities affecting the data. The program link NRMSOL solves the normal equations by two distinct methods: (1) by a stepwise-regression technique, and (2) by a singular value decomposition method. The Gauss-Jordan elimination technique (Efroymson, 1960) is employed in the stepwise regression routine. To employ the singular value analysis (Hanson and Lawson, 1969), which is a very powerful tool in rank-deficient situations, the normal equations are first modified by the Choleski square-root transformation (Hartree, 1961) before undergoing the Householder orthonormal transformations which are the core of the singular value analysis.

The output from NRMSOL consists of an improved set of initial conditions for ephemeris-related quantities and astronomical constants. If the problem under consideration is rank-deficient, then one would employ the results of the singular value analysis; otherwise, the results of either the Gauss-Jordan inversion or the full-rank solution may be adopted. The results may then be used as new initial conditions for PLINT and the process repeated until convergence. Plots of the residuals of the data before improving the nominal values may be obtained by use of PLOR, while plots of the linearized residuals (the predicted residuals after improving the various parameters) may be obtained from the programs LSQR and PLOR.

\section{Observational Data}

Optical observations from 1910 to 1969 of the major planets, except Pluto, have been collected from publications of the United States Naval Observatory (USNO), (Watts and Adams, 1949; Hammond and Watts, 1927; Morgan, 1933; Watts et al. 1952; Adams et al., 1964; Adams and Scott, 1964, 1965, 1967a, b, 1968, 1969; Morgan and 
TABLE IA

Transit circle observations

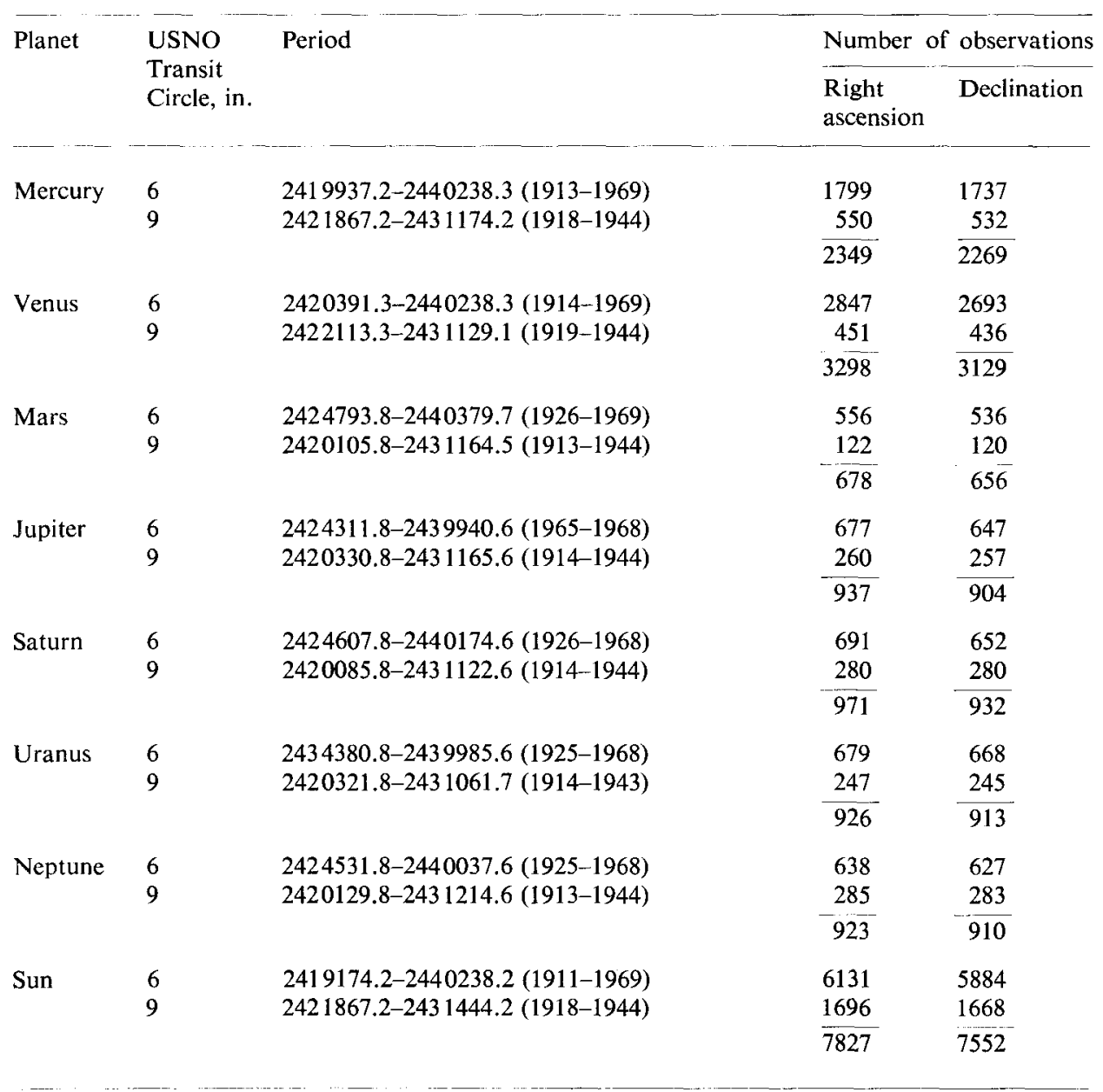

Scott, 1948; Klock and Scott, 1970). The observations made with the 6-in. and 9-in. Transit Circles at the USNO, Washington, D.C., are summarized in Table IA.

The distribution of the data is depicted in Figure 2 which is a plot of the planetary (and solar) declinations vs right ascension. The data obviously cluster close to the ecliptic plane, and in Figure 3 the excursion in declination from the ecliptic is plotted vs right ascension. The very predominant grouping on the ecliptic reflects the great number of solar observations.

The collection of the data over the period 1911-49 was made by C. Oesterwinter of the U.S. Naval Weapon Laboratory (NWL), Dahlgren, Virginia. The remaining collection has been the joint effort of the USNO, NWL, and JPL. All of these data have been punched on cards in a standard format (O'Handley, 1968). 


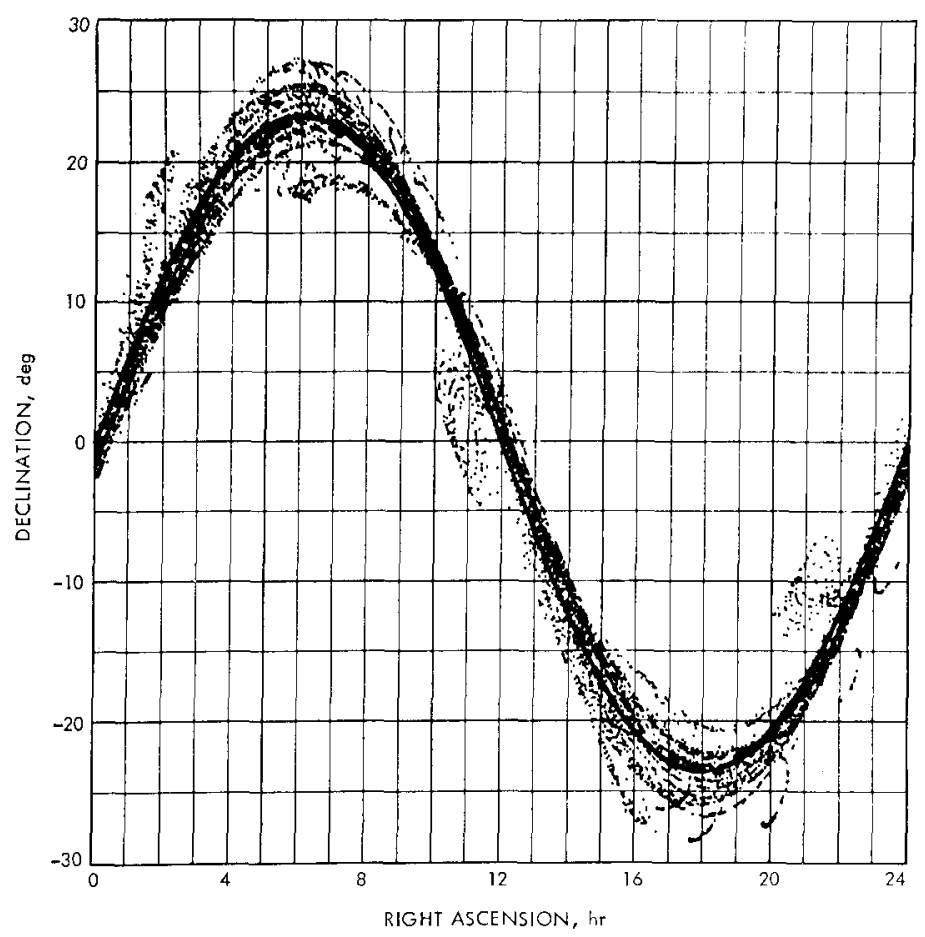

Fig. 2. Right ascension vs declination of the optical observations.

The 1071 right ascension and declination observations of Pluto have been obtained from the paper by Cohen, Hubbard, and Oesterwinter (1967). They are photographic observations which cover the period 1914 to 1965 . The six sources for observations are listed in Table IB. The final optical data set contains 36245 observations at this time.

Planetary radar range data have taken since 1961 by JPL and the Massachusetts Institute of Technology (MIT). The early data prior to 1964 are primarily of historical value since the accuracy of these data contributes little or nothing to the solution of the elements and radii of the inner planets.

TABLE IB

Photographic observations of Pluto

Pre-discovery

Sharaf-Bodnikova

Yerkes-McDonald

Lowell

Yerkes

Halliday
14 observations

33 observations

183 observations

310 observations

21 observations

9 observations
1914-1927

$1930-1958$

1930-1953

$1930-1951$

1962-1963

1963-1965 


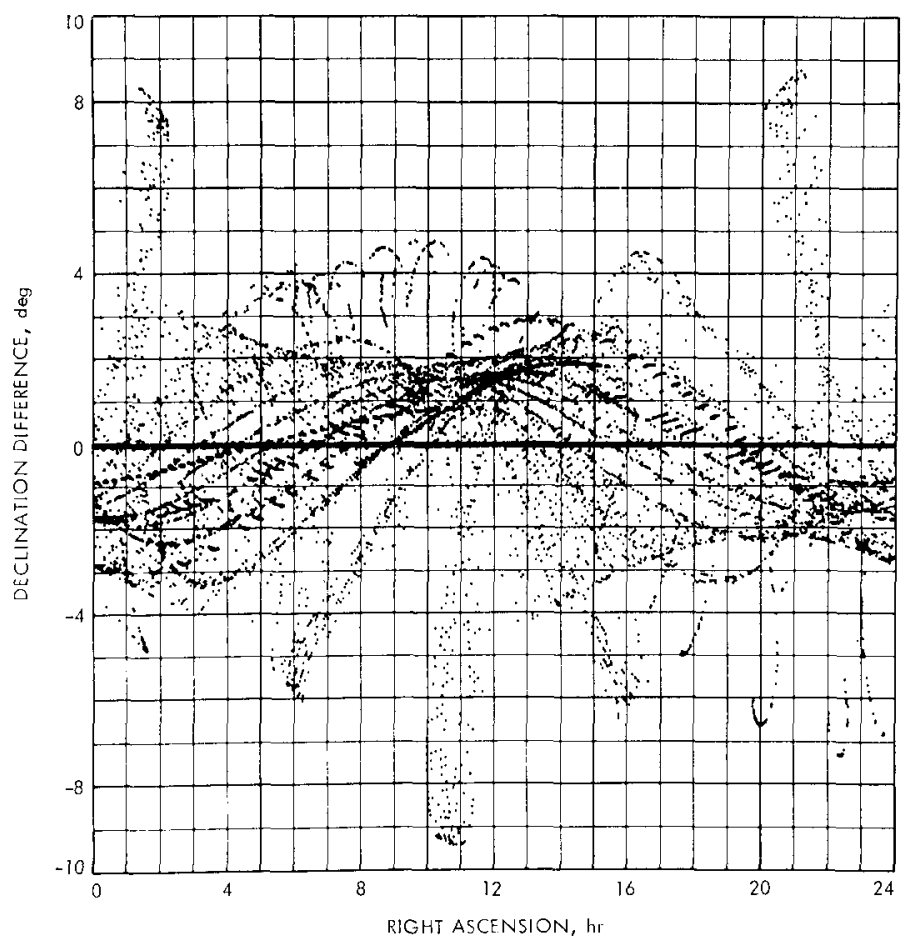

Fig. 3. Declination difference vs right ascension of the optical observations.

Since 1964 a radar data set of over 1000 precise measurements has been collected for the planets Mercury, Venus, and Mars. These observations have been made at the Arecibo (Puerto Rico) Ionospheric Observatory (AIO) of the Cornell-Sydney University Astronomy Center, the Millstone and Haystack sites of MIT, and DSS-13 (Venus Antenna) and DSS-14 (Mars Antenna) of the NASA/JPL Goldstone (Calif.) Tracking Facility.

Current ephemerides can predict the position of Venus to better than $20 \mu$ s in range. It has been found that the inclusion of AIO data degrades this ephemeris. A systematic bias of approximately $30 \mu$ s appears in the residuals. Consequently, most AIO data have been removed from the current data set. These observations have been re-examined and will be incorporated into future solutions.

The initial 9 AIO observations of Mars have been removed because of residuals close to $1 \mathrm{~ms}$ or larger. The remaining 30 range points are employed with their published weights and are retained in the solution. In 1967 the Haystack radar antenna made 148 radar range measurements of Mars over an 8-week interval from April 7 to June 2 on ten separate nights (Pettengill et al., 1969). There was sufficient sensitivity to measure for the first time, in terms of round-trip echo delay, the variations in elevation of the Martian surface as the planet rotated on its axis.

From May 7 to August 16, 1969, 236 precise bistatic range measurements and 
4 monostatic range measurements were made at the Goldstone Tracking Facility of JPL. The bistatic experiment consisted of using two antennas at the Goldstone Tracking Station of JPL. The Venus $25.9-\mathrm{m}$ antenna with a $450-\mathrm{kW}$ transmitter was used to transmit a signal to the planet, and the Mars $64-\mathrm{m}$ antenna was employed as the receiver. The monostatic measurements made use of the Venus antenna alone. These data are to be published in the near future.

The range measurements of Mars taken at MIT in 1967 have a nominal 'delay error' of $\pm 20 \mu \mathrm{s}$, and the range measurements of Mars made in 1969 at JPL are of 1, 2, and $3 \mu \mathrm{s}$ precision. The intent at this time is to minimize the effects of topography and correct the orbital elements and radius. The topography imbedded in the range measurements amounts to $80 \mu$ s of time-delay in addition to the error in the ephemeris. As a result, the data points were downweighted individually by a value which reflects the original quoted error and the number of data points taken. This factor was 2.2 for MIT and 8 for JPL.

A summary of the complete radar data set used in this solution is given in Table II. These data are available in a uniform format on punched cards (O'Handley, 1968) and are obtained from publications listed in Evans et al., 1965; Evans, 1966; Dyce and Pettengill, 1967; Pettengill et al., 1967; Muhleman et al., 1968; Goldstein, 1968; Private Communication, 1967, 1968.

The collection of these data has been a joint effort by MIT-Lincoln Laboratory and JPL.

Spacecraft tracking data represent a third type of data utilized in this investigation. Six tracking stations of NASA/JPL Deep-Space Tracking Stations have been employed

TABLE II

Radar range observations

Data source $\quad$ Period

Mercury

AIO

2438493.2-2439363.2 (1964-1966)

119

Haystack (MIT)

2439425.3-2440064.2 (1966-1968)

88

Summary 1964-1968

Number of

observations

Venus

Haystack (MIT)

2439161.3-2440063.2 (1967-1968)

$2438541.2-2439791.3(1964-1967)$

JPL (Venus DSS)

2438447.0-2439725.2 (1964-1967)

Summary 1964-1968

207

\section{Mars}

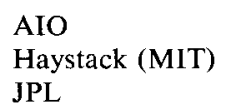

2438792.8-2438915.5 (1964-1965)

2439587.7-2439643.5 (1967)

2430348.5-2440449.7 (1969)

Summary 1964-1969 
TABLE III

Mariner 5 tracking data

\begin{tabular}{|c|c|c|c|}
\hline Station & Period & $\begin{array}{l}\text { No. of } \\
\text { observations }\end{array}$ & Type \\
\hline $\begin{array}{l}\text { Mars-Goldstone, Calif. } \\
\text { DSS-14 }\end{array}$ & Oct. 14,1967 -Oct. 22,1967 & 58 & Range \\
\hline $\begin{array}{l}\text { Echo-Goldstone, Calif. } \\
\text { DSS-12 }\end{array}$ & Oct. $15,1967-$ Oct. 25,1967 & 39 & Doppler \\
\hline $\begin{array}{l}\text { Mars-Goldstone, Calif. } \\
\text { DSS-14 }\end{array}$ & Oct. 14,1967 -Oct. 22,1967 & 43 & Doppler \\
\hline $\begin{array}{l}\text { Woomera, Australia } \\
\text { DSS- } 41\end{array}$ & Oct. 15,1967 -Oct. 25,1967 & 74 & Doppler \\
\hline $\begin{array}{l}\text { Tidbinbilla, Australia } \\
\text { DSS-42 }\end{array}$ & Oct. 18,1967 -Oct. 19,1967 & 5 & Doppler \\
\hline $\begin{array}{l}\text { Johannesburg, S. Africa } \\
\text { DSS-51 }\end{array}$ & Oct. $22,1967-$ Oct. 22,1967 & 5 & Doppler \\
\hline $\begin{array}{l}\text { Cebreros, Spain } \\
\text { DSS-62 }\end{array}$ & Oct. 15,1967 -Oct. 25,1967 & 59 & Doppler \\
\hline
\end{tabular}

Note: Encounter of the Mariner 5 spacecraft took place on Oct. 19, 1967.

to obtain a reduced set of 225 Doppler measurements of Mariner 5 during the encounter phase of that mission. In addition to Doppler data, 58 range measurements from the Mars antenna at the Goldstone Tracking Facility have been utilized (see Table III). These data were obtained by means of ranging equipment designed by R. C. Tausworthe of JPL (Tausworthe, 1967). Although the ranging system is capable of achieving a measurement accuracy of better than $\pm 0.1 \mu \mathrm{s}$, a standard deviation of $0.3 \mu \mathrm{s}$ has been used in weighting the spacecraft range data employed in the solutions presented in this paper.

A standard deviation of $0.015 \mathrm{~Hz}$ has been used for the Doppler measurements.

\section{Results}

The data have been analyzed for corrections to the planetary orbital elements and several astronomical constants. The nominal values employed in the integration are taken from Development Ephemeris 69 (O'Handley et al., 1969), and the relevant astronomical constants are listed in Table IV. To demonstrate more clearly some of the problems which must be examined in greater detail in the future, we present six sets of solutions based upon three different combinations of data: (1) optical, (2) optical and radar, (3) optical, radar, and Mariner 5 radio tracking data.

A summary of the solutions is presented in Table $\mathrm{V}$ which contains only the results for the planetary masses and radii. The table contains the data type(s), number of data points, number of estimated parameters and values for the inverse planetary masses, 
together with the astronomical unit and radii of the inner planets. In addition to the parameters given in the table, six elements for each planet were estimated together with limb biases in right ascension and declination for Mercury and Venus.

Solution 1 results from analyzing only the optical data and estimating all the planetary masses, while in Solution 2 the masses of Venus, Earth-Moon, and Mars remain fixed at their nominal values (as determined from Ranger and Mariner spacecraft data).

TABLE IV

\begin{tabular}{lc}
\multicolumn{2}{c}{ Nominal values of astronomical constants } \\
\hline Speed of light & $299792.5 \mathrm{~km} / \mathrm{s}$ \\
Astronomical unit & $149597893 \mathrm{~km}$ \\
Inverse masses: & \\
Sun & 1 \\
Mercury & 5983000 \\
Venus & 408522 \\
Earth and Moon & 328900.1 \\
Mars & 3098700 \\
Jupiter & 1047.3908 \\
Saturn & 3499.2 \\
Uranus & 22930 \\
Neptune & 19260 \\
Pluto & 1812000 \\
Earth/Moon & 81.301 \\
&
\end{tabular}

Solutions 3 and 4 differ from 1 and 2, respectively, by the inclusion of radar data and the associated parameters of the radii and the AU. Solutions 5 and 6 represent another combination, with the inclusion of the Mariner 5 spacecraft data and the associated additional spacecraft parameters in the solution.

\section{Discussion}

Perusal of the results listed in Table V will amply demonstrate several salient features: (1) the over-optimism of the formal statistics relative to the disparity among several solutions involving different data types, (2) the relative power of radar and radiotracking data vis-à-vis purely optical data, and (3) the problems which could arise by adopting results based upon a single type of data.

The solution for the mass of Mercury obviously depends upon the types of data analyzed and is strongly influenced by the weighting and distribution of the radar data. The values for the reciprocal mass of Venus are significant since they result from solutions which contain varied types of data including the Mariner 5 encounter data. The value of 408523.8 is in good agreement with the value reported by Anderson and Efron (1969) of 408521.8 \pm 1.0 from their analysis of the 1962 Mariner 2 and 1967 Mariner 5 data. The values for the reciprocal mass of Mars in Solutions 1, 3, and 5 , although relatively indeterminable, are in good agreement with the nominal value of 


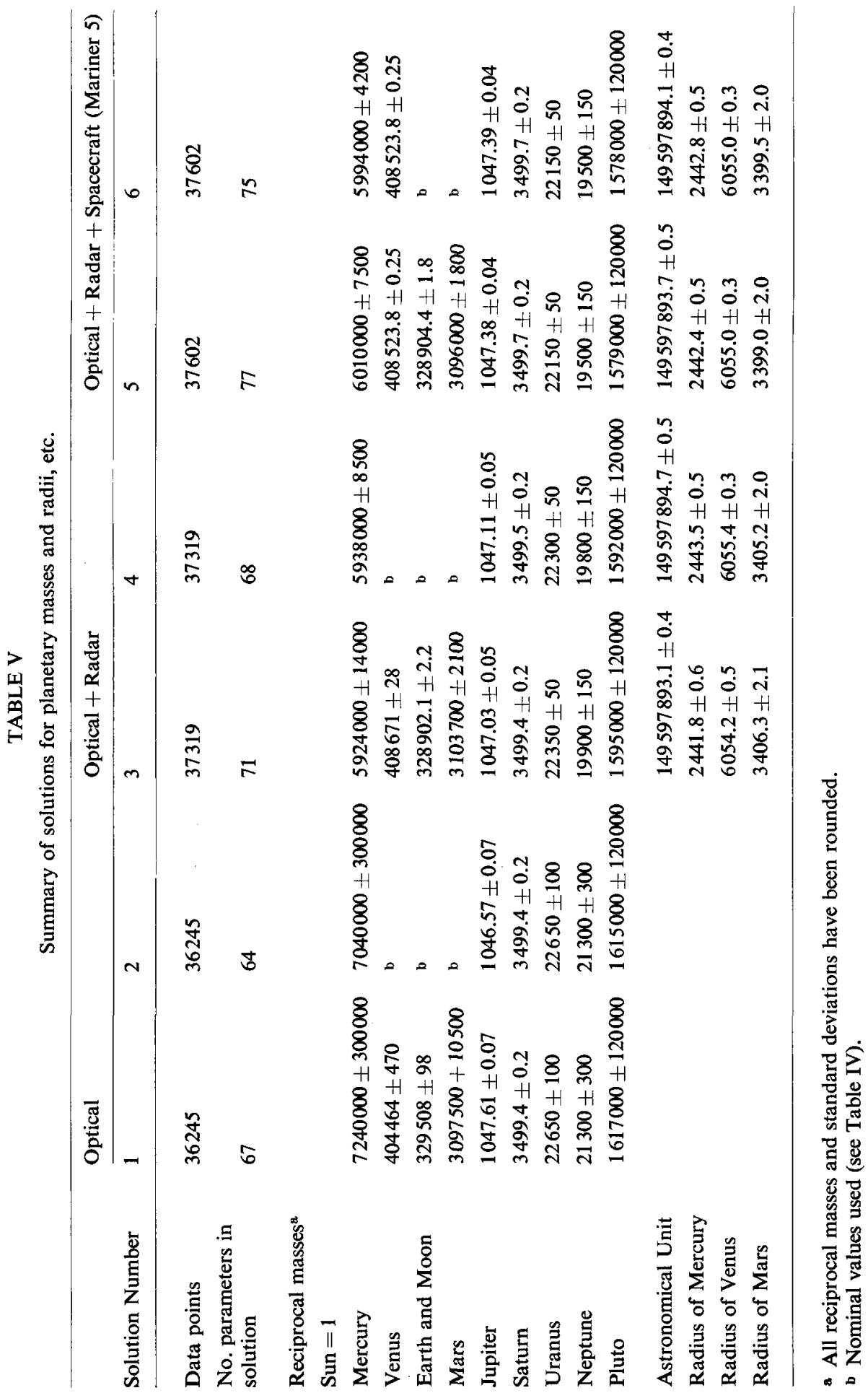


$3098714 \pm 5$ which was obtained by Null (1969) in his analysis of 1964 Mariner 4 Doppler data taken during enounter. Whereas JPL solutions for planetary masses have in the past employed only tracking data, the treatment of this paper introduces a solution which is gravitationally consistent. The mass of Uranus is altered a great deal (relative to its formal error) by the introduction of radar data.

The mass of Pluto is remarkably stable, at least with the data currently being analyzed. However, the result may be affected by poorly understood trends as suggested by Duncombe et al. (1968).

Before a more definitive set of planetary masses can be obtained by gravitationally consistent methods, the introduction of other radio-tracking data must be made. The question of weighting and distribution of data, together with a thorough analysis of the effects of errors in precession, equinoxial motion, obliquity rate, and errors in star catalogues, should be investigated. The present results should be useful, however, in estimating some realistic bounds on planetary masses. At this time we would recommend that the masses of Solution 6 be used together with the spacecraft-determined values for the masses of the Earth and Moon and Mars.

\section{Acknowledgments}

The system of computer programs used in this research effort was formulated by Dr Charles L. Lawson of JPL. The system analysis and coding for the Univac 1108 was carried out by the KMS Technology Center.

Joyce Steinberg, Steve Ritchie, and KMS Industries, Inc. are singled out for their interest in the successful implementation of the system of programs to improve the knowledge of the locations of planets in the solar system.

George Null is responsible for the segments of SSDPS which allowed us to process Pluto observations and implementation of the series into VARSER. Roger Broucke's work with the Chebyshev and Fourier series made the solutions for planetary masses possible. We are indebted to J. D. Anderson for his comments in the preparation of the manuscript.

\section{References}

Adams, A. N. and Scott, D. K.: 1964, 'Observations of the Sun, Moon, and Planets; Six-Inch Transit Circle Results, March 8, 1963-July 2, 1964', U.S. Naval Obs., Washington, Circular No. 105.

Adams, A. N. and Scott, D. K.: 1965, 'Observations of the Sun, Moon, and Planets; Six-Inch Transit Circle Results, July 7, 1964-Dec. 24, 1964; U.S. Naval Obs., Washington, Circular No. 108.

Adams, A. N. and Scott, D. K.: 1967a, 'Observations of the Sun, Moon, and Planets; Six-Inch Transit Circle Results, Jan. 11, 1965-April 11, 1966', U.S. Naval Obs. Washington. Circular No. 115.

Adams, A. N. and Scott, D. K.: 1967b, 'Observations of the Sun, Moon, and Planets; Six-Inch Transit Circle Results May 29, 1966-July 10, 1967', U.S. Naval Obs. Washington. Circular No. 118.

Adams, A. N. and Scott, D. K.: 1968, Publ. U.S. Naval Obs. Washington, Second Series, Vol. 19, 333-80.

Adams, A. N. and Scott, D. K.: 1969, 'Observations of the Sun, Moon, and Planets; Six-Inch Transit Circle Results, July 12, 1967-June 25, 1968', U.S. Naval Obs. Washington Circular No. 124. 
Adams, A. N., Bestal, S. M., and Scott, D. K.: 1964, Publ. U.S. Naval Obs., Washington, Second Series, Vol. 19, 47-110.

Anderson, J. D. and Efron, L.: 1969, Bull. Am. Astron. Soc. 1, 231.

Broucke, R.: 1969, Celes. Mech. 1, 110.

Broucke, R. and Garthwaite, K.: 1969, Celest. Mech. 1, 271.

Cohen, Charles J., Hubbard, Elbert C., and Oesterwinter, C.: 1967, Astron. J. 72, 973.

Duncombe, R. L., Klepczynski, W. J., and Seidelmann, P. K.: 1968, Astron. J. 73, 830.

Dyce, R. B. and Pettengill, G. H.: 1967, Astron. J. 72, 771.

Eckert, W. J. and Brouwer, D.: 1937, Astron. J. 46, 125; errata 47, 83.

Eddington, A. S.: 1960, The Mathematical Theory of Relativity, Cambridge Univ. Press, New York, 2nd edition, p. 93.

Efroymson, M. A.: 1960, 'Multiple Regression Analysis', Chap. 17 in Math. Methods for Digital Computers, Vol. I (ed. by A. Ralston and H. S. Wilf), John Wiley, N.Y., p. 191.

Evans, J. V. et al.: 1965, Astron. J. 70, 486.

Evans, J. V. et al.: 1966, Astron. J. 71, 897.

Goldstein, R. M.: 1968, Astron. J. 73, 829.

Hammond, J. C. and Watts, C. B.: 1927, Publ. U.S. Naval Obs. Washington, Second Series, Vol. 11, pp. 153-79.

Hanson, R. J. and Lawson, C. L.: 1969, Math. Comp. 23, 787.

Hartree, D. R.: 1961, Numerical Analysis, Clarendon Press, Oxford, p. 180.

Klock, B. L. and Scott, D. K.: 1970, 'Observations of the Sun, Moon, and Planets; Six-Inch Transit Circle Results, June 30, 1968-July 17, 1969', U.S. Naval Obs. Washington. Circular No. 127.

Melbourne, W. G.: 1970, 'The Determination of Planetary Masses from Radio Tracking of Space Probes and Planetary Radar' in Dynamics of Satellites (1969) (ed. by B. Morando), Springer Verlag, Berlin, p. 63.

Morgan, H. R.: 1933, Publ. U. S. Naval Obs. Washington, Second Series, Vol. 13, 101-55.

Morgan, H. R. and Scott, F. P.: 1948, Publ. U.S. Naval Obs. Washington, Second Series, Vol. 15.

Muhleman, D. O. et al.: 1968, JPL Radar Range and Doppler Observations of Venus, 1961-1966, Technical Report 32-1123, Jet Propulsion Laboratory, Pasadena, Calif., July 1.

Null, G. W.: 1969, Bull. Am. Astron. Soc. 1, 356.

O'Handley, D. A.: 1968, Card Format for Optical and Radar Planetary Data, Technical Report 32-1296, Jet Propulsion Laboratory, Pasadena, Calif.

O'Handley, D. A., Holdridge, D. B., Melbourne, W. G., and Mulholland, J. D.: 1969, JPL Development Ephemeris 69, Technical Report 32-1465, Jet Propulsion Laboratory, Pasadena, Calif. Pettengill, G. H., Dyce, R. B., and Campbell, D. B.: 1967, Astron. J. 72, 330.

Pettengill, G. H. et al.: 1969, Astron. J. 74, 461.

Private Communication: 1967, Millstone and Haystack Observatories, '1967 Venus Observations', Smith, W., Lincoln Laboratory with Melbourne, W. G., JPL, October.

Private Communication: 1968, Haystack Observatory, '1967-1968 Mercury Observations', Shapiro, I. I., MIT with O’Handley, D. A., JPL, October 28.

Robertson, H. P.: 1962, Space Age Astronomy (ed. by A. J. Deutsch and W. E. Klemperer), Academic Press, N.Y., p. 228.

Tausworthe, R. C.: 1967, 'Ranging the 1967 Mariner to Venus', Paper No. 36.4, 1967, IEEE International Conf. Record, New York, March 20-23.

Watts, C. B. and Adams, A. N.: 1949, Publ. U.S. Naval Obs. Washington, Second Series, Vol. 16, 59-203.

Watts, C. B., Scott, F. P., and Adams, A. N.: 1952, Publ. U.S. Naval Obs. Washington, Second Series, Vol. 16, 395-445. 